\title{
Модуляционно-поляризационная диагностика термонапряжений в интегральном преобразователе давления
}

\author{
(C) И.В. Михайленко ${ }^{1}$, А.Т. Орлов ${ }^{2}$, Б.К. Сердега ${ }^{1, \uparrow}$ \\ ${ }^{1}$ Институт фризики полупроводников им. В.Е. Лашкарева Национальной академии наук Украины, \\ 03028 Киев, Украина \\ ${ }^{2}$ Национальный технический университет Украины „Киевский политехнический институт“, \\ 03057 Киев, Украина \\ IE-mail: bserdega@isp.kiev.ua
}

(Получена 6 сентября 2016 г. Принята к печати 26 сентября 2016 г.)

В полупроводниковом преобразователе давления, состоящем из сенсорного резистора, имплантированного в кремниевую мембрану, как упругий элемент механики, техникой модуляционной поляриметрии детектированы внутренние механические напряжения. Координатные распределения одноосных напряжений измерены в двух случаях - напряжения, остаточные от локальных неоднородностей легирования кристалла, и напряжения, обусловленные разогревом от протекающего тока. Координатное распределение температуры от теплового потока, выделяемого током в резисторе, получено двойным интегрированием функции напряжения с учетом соответствующих граничных условий.

DOI: 10.21883/FTP.2017.04.44346.8311

\section{1. Введение}

Микроэлектронные сенсоры давления, производство которых базируется на технологии кремниевой микроэлектроники, находят широкое применение во многих отраслях промышленности [1]. Технологии проектирования и изготовления сенсоров хорошо отработаны, о чем свидетельствует факт их серийного производства [2]. Однако степень искажения измеряемой величины „паразитными“ механическими напряжениями в чувствительном элементе, не связанными непосредственно с измеряемым параметром, исследована недостаточно. Известно несколько причин их возникновения, в частности асимметрия размеров сенсора, особенности структуры кристаллической ячейки кремния, тип и количество легирующего материала, градиенты легирования кристалла и/или его слоистая структура, в том числе наличие пленки $\mathrm{SiO}_{2}$, а также локальные термомеханические эффекты, вызванные электропитанием преобразователя. Во всех этих случаях величина механического напряжения является равнодействующей всех сил и, хотя она редко превышает предел прочности кристалла, ее влияние на такие параметры датчика, как надежность, линейность характеристики преобразования, величина обратного тока $p-n$-перехода, может быть существенным. Вот почему сведения о локализации напряжений, о соотношении компонент напряжений и их эволюции представляют как физический, так и технологический интерес. Получение такой информации является главной целью данной работы. Вторичная цель состоит в испытании потенциальных возможностей модуляционной поляриметрии (МП) на обнаружительную способность относительно величины оптической анизотропии в объекте, в котором применение других методов (рентгеновский, комбинационное рассеяние, классический оптико-поляризационный) ввиду его микроэлектронного исполнения становится проблематичным.

\section{2. Методика эксперимента}

Образцом для исследований служил чувствительный элемент датчика давления (чип) с размерами $(x \times z \times y)$ $6 \times 6 \times 0.4$ мм, изготовленный по групповой технологии из серийной кремниевой пластины $n$-типа проводимости (удельное сопротивление $\rho=1 \mathrm{OM} \cdot \mathrm{cm}$ ) с последующим разделением ее на элементы. Тензорезистор изготавливался имплантацией бора на глубину 2.5 мкм в центре элемента с размерами $200 \times 100$ мкм и имел сопротивление $\sim 900$ Ом. Чип объединяет в себе две функции: упругого элемента механики в виде профилированной мембраны, воспринимающей распределенное давление внешней среды, и интегрированного в ее центре резисторного сенсора. Действие последнего основано на эффекте поперечной тензоэдс [3-5], возникающей в одноосно-деформированном ограниченном кристалле при протекании в нем тока. Методом анизотропного травления [6] мембране придана структура в форме двух утолщенных островков (рис. 1,a) [7], посредством которых ее деформация в плоскости от гидростатического давления преобразуется в локальное одноосное растяжение в центре, в месте расположения сенсора.

Сложный профиль мембраны при малых ее размеpax ограничивает выбор методов исследования ее напряженного состояния. Поэтому плодотворной может оказаться методика, основанная на регистрации изменения состояния поляризации излучения, отраженного от плоской поверхности чувствительного элемента. Здесь используется тот общеизвестный факт, что отражающей толщиной кристалла является длина поглощения (затухания) интенсивности излучения. В данном случае оптическая индикатриса (эллипсоид Френеля) своими осями $x, z$ совпадает с поверхностью чипа. Предполагается, что эта анизотропия является макроскопической вследствие того, что формируется пониженной до второго порядка симметрией профиля мембраны. Что же 

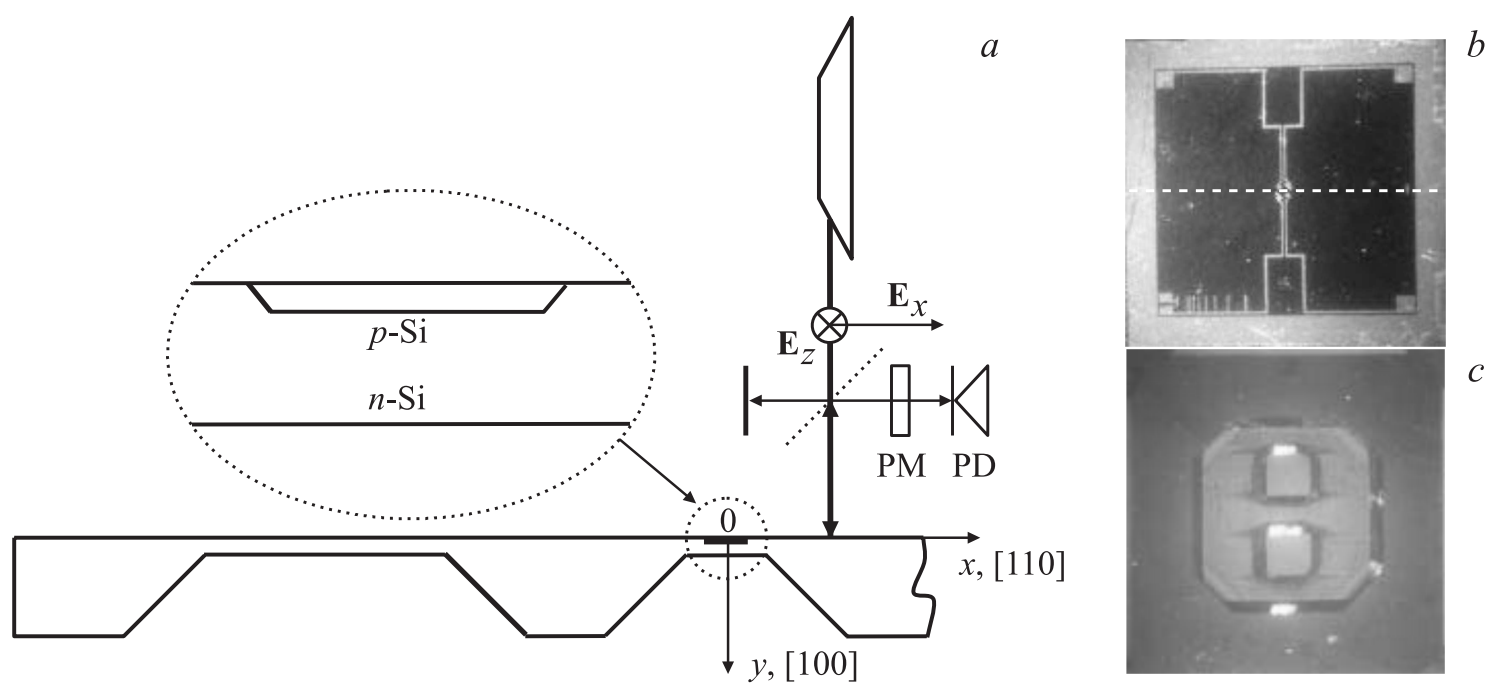

Рис. 1. Геометрия опыта, кристаллографическая и координатная ориентации структуры датчика в поперечном разрезе $(a)$; лицевая $(b)$ и обратная $(c)$ стороны чипа. Ориентация поля падающей на образец волны $\mathbf{E}=\mathbf{E}_{x}+\mathbf{E}_{z}$. РМ - модулятор поляризации, $\mathrm{PD}$ - фотодетектор.

касается напряжения, связанного с дивергенцией градиента легирования $p-n$-перехода, отделяющего сенсор от мембраны, то его плоская деформация в пределах контура сенсора не создает анизотропии. Однако форма сенсора в виде прямоугольника с учетом суммирования величин напряжений на его границах все же может быть причиной одноосной деформации, компонента которой имеет направление, совпадающее с макроскопической деформацией мембраны. Поэтому азимут электрического поля $\mathbf{E}$ волны падающего поляризованного излучения как сумма компонент $\mathbf{E}_{x}$ и $\mathbf{E}_{z}$ (рис. 1,a) устанавливался, согласно [8], под углом $45^{\circ}$ к осям образца. Перемещением образца вдоль координаты $x$ (штриховая линия на рис. $1, b)$ осуществлялось сканирование его поверхности сфокусированным лазерным излучением. Наличие анизотропии в отражающей среде приводит к возникновению в отраженном луче разности фаз $\delta=\varphi_{x}-\varphi_{z}$ между ортогональными компонентами излучения $\mathbf{E}_{x}$ и $\mathbf{E}_{z}$. В результате происходит преобразование линейной поляризации в общем случае в эллиптическую, в составе которой циркулярная компонента является мерой деформации. Величина анизотропии определяется действительной и мнимой частями комплексного показателя преломления $n_{x}=n_{x}^{\prime}+i n_{x}^{\prime \prime}=\sqrt{\varepsilon_{x}}, n_{z}=n_{z}^{\prime}+i n_{z}^{\prime \prime}=\sqrt{\varepsilon_{z}}$ и имеет вид $[8]$

$$
\begin{aligned}
\delta= & \operatorname{arctg}\left[\frac{2 n_{y}^{\prime} n_{y}^{\prime \prime}}{1-\left(n_{z}^{\prime}\right)^{2}-\left(n_{z}^{\prime} n_{z}^{\prime \prime}\right)^{2}}\right] \\
& -\operatorname{arctg}\left[\frac{2 n_{x}^{\prime} n_{x}^{\prime \prime}}{1-\left(n_{x}^{\prime}\right)^{2}-\left(n_{x}^{\prime} n_{x}^{\prime \prime}\right)^{2}}\right],
\end{aligned}
$$

где

$$
\begin{array}{cc}
n_{x}^{\prime}=n^{\prime}+C_{\mathrm{br}} X, & n_{x}^{\prime \prime}=n^{\prime \prime}+C_{\mathrm{br}} X, \\
n_{z}^{\prime}=n^{\prime}-v C_{\mathrm{br}} X, & n_{z}^{\prime \prime}=n^{\prime \prime}-v C_{\mathrm{br}} X
\end{array}
$$

обозначают размеры эллипсоида Френеля и их деформационную эволюцию в модели закона Гука, $v$ - коэффициент Пуассона, $C_{\mathrm{br}}-$ константа Брюстера, $X=\sigma_{x}-\sigma_{z}-$ разность главных компонент механического напряжения в $x-z$-плоскости в точке зондирования.

Для экспериментального измерения внутреннего напряжения использовался оптико-поляризационный метод, модифицированный применением модуляции поляризации зондирующего излучения [9]. Его детальное описание приведено в [10], поэтому здесь ограничимся лишь изложением принципа измерения. По аналогии с эллипсометрией его можно назвать нуль-методом на том основании, что образец облучается линейно поляризованным излучением в исходном состоянии, а в отражении регистрируется циркулярная $V$-компонента (в обозначениях [11]), отсутствующая в составе падающего излучения. Ее величина определяется произведением интенсивностей компонент линейно поляризованной волны и равна $V=E_{x}^{2} E_{z}^{2} \sin \delta \sin \omega t$. Здесь $\omega$ - частота модуляции, а $\delta$ - разность фаз между ортогональными компонентами поля волны $\mathbf{E}_{x}$ и $\mathbf{E}_{y}$, определяемая величиной усилия сжатия/растяжения: $\delta \propto\left(n_{x}-n_{z}\right) X$. Оптическая схема представляла собой поляриметрический интерферометр Майкельсона, в котором изменение поляризации излучения, отраженного по нормали от образца, сравнивается с фиксированным состоянием поляризации излучения в опорном плече. Модуляционный поляриметр совместно с присоединенным к нему линейным поляризатором играет роль анализатора, с помощью которого сигнал фотодетектора от циркулярной компоненты отделялся от сигнала в общем случае эллиптически поляризованного излучения. Кроме того, техникой МП исключается из измеряемого сигнала постоянная во времени компонента от неполяризованного зондирующего излучения, что является дополнительным аргументом нуль-метода. 
Регистрация $V$-компоненты осуществлялась $\mathrm{Si}$-фотодетектором, сигнал которого усиливается lock-in-нановольтметром. Наличие опорных состояния поляризации и сигнала фазы модуляционного напряжения модулятора (частота $\omega=50$ кГц) позволяли регистрировать как величину деформации, так и ее знак.

Источником линейно поляризованного излучения для зондирования образца использован гелий-неоновый лазер (длина волны $\lambda=0.63$ мкм, коэффициент поглощения в $\left.\mathrm{Si} \alpha=4.5 \cdot 10^{3} \mathrm{~cm}^{-1}\right)$. Одномодовый режим лазера и высококачественная фокусирующая оптика позволяли собирать излучение в световое пятно на образце размером $\sim 10$ мкм, что обеспечивало разрешающую способность измерения напряжения на размере сенсорного элемента. В недеформированном элементе измерялась величина $V(x)$ в двух случаях - в условиях равновесия образца при комнатной температуре, а также при номинальной величине напряжения питания $3 \mathrm{~B}$. В обоих случаях измеренный сигнал нормировался на интенсивность координатного распределения немодулированного излучения во избежание проявления вклада от неоднородностей изотропного отражения. Калибровка деформационной чувствительности измерительной системы проводилась измерением фазового сдвига между ортогональными компонентами излучения в эквивалентной кремниевой пластинке, двулучепреломление в которой было вызвано внешним контролируемым усилием. Для исключения тензорных эффектов (деформационного и оптико-поляризационного) образец для калибровки выбирался с той же кристаллографической ориентацией.

\section{3. Результаты измерений и обсуждение}

Сложная функциональная связь в уравнении (1) между величинами внутреннего напряжения и фазового сдвига диктует необходимость в определении метрологической способности характеристики $\delta(X)$ с точки зрения степени ее нелинейности. Ее графическое выражение, полученное при значениях оптических параметров, свойственных кристаллу кремния, имеет вид кривой на рис. 2. Действительно, представленная характеристика, будучи суммой двух различающихся параметрами тригонометрических функций, является даже более сложной, чем каждая из них в отдельности. Происхождение и эволюция особенностей этой характеристики представляет отдельный интерес исследования. В нашем случае достаточно того, что в ней имеется протяженный линейный участок (на вставке к рис. 2), упрощающий калибровку чувствительности дополнительным измерением. Этим действием устанавливается соответствие величины (отмечено точкой на вставке) фазовой задержки $\delta_{V}(X)$ в выражении для циркулярной $V$-компоненты и напряжения от приложенного усилия $X$ сжатия.

В свободном от закрепления в корпус чувствительном элементе наличие деформации (механического напряжения) может быть обусловлено несколькими причинами.

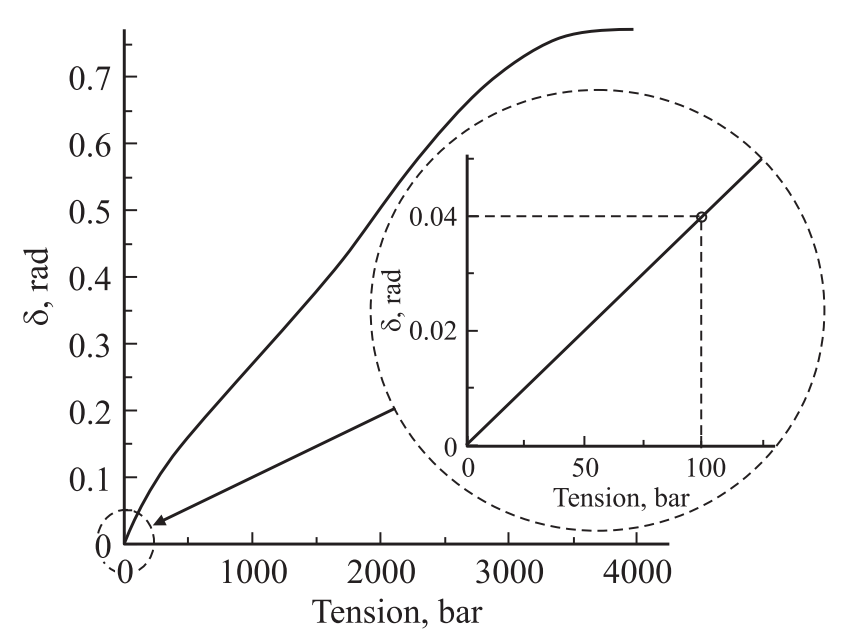

Рис. 2. Расчетная зависимость фазового сдвига между ортогональными компонентами линейно поляризованного излучения, отраженного от одноосно деформированного образца кремния. Точкой обозначена величина фазового сдвига, полученная на эквивалентном образце, сжатом контролируемым усилием. В расчете использованы исходные значения коэффициента экстинкции 0.004 , показателя преломления $3.8, v=0.4$, $C=0.0007 \kappa \Gamma / \mathrm{cm}^{2}$.

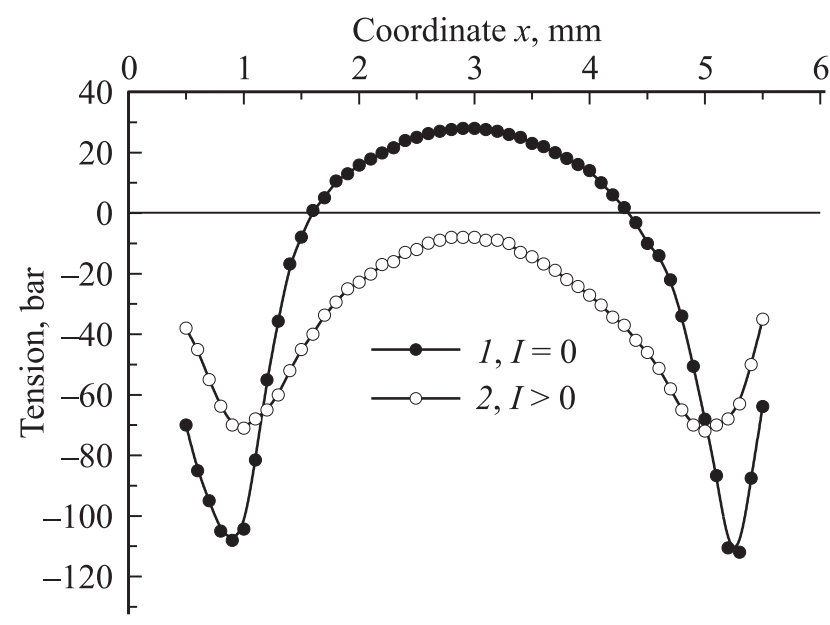

Рис. 3. Координатные распределения разностей главных значений механических напряжений $\sigma_{x}-\sigma_{z}$ в равновесии и в условиях локального нагрева энергией $10^{-2}$ Дж от протекающего в тезорезисторе тока от внешнего источника $I=3 \cdot 10^{-3} \mathrm{~A}$.

К ним относятся неоднородный профиль мембраны и связанная с ним компонента градиента поверхностного натяжения, неоднородное легирование кристалла при создании преобразователя, а также неоднородный градиент температуры вследствие выделения тепла от протекающего электрического тока при работе преобразователя. Эффекты, вызванные первыми двумя факторами, являются аддитивными и изображаются кривой 1 на рис. 3 , полученной сканированием луча вдоль оси $x$ по средней линии мембраны. Отметим прежде всего, что чип лишен симметрии формы четвертого порядка вследствие той структуры, которая заключена в наличии продольных 
утолщений в центре. Именно по этой причине одноосная компонента деформации в центральной части мембраны не равна нулю (кривая 1 ). Этим обстоятельством симметрия мембраны понижена до второго порядка, что привело к появлению некомпенсированной разности величин $\sigma_{x}-\sigma_{z} \neq 0$. Вместе с тем выделение тепла в центре образца от протекающего тока в сенсоре микрометрового размера как раз является источником радиального теплового потока и связанного с ним симметричного распределения напряжения. Именно этот результат показан на рис. 3, на котором кривая 2 функции $\left(\sigma_{x}-\sigma_{z}\right)(x)$ стремится к 0 при $x=3$. В связи с этим уместно отметить, что однозначный характер этой кривой не нарушает условие неподвижности образца, поскольку примерно такое же распределение напряжения наблюдается вдоль координаты $z$ как результат равенства нулю главного вектора всех действующих на образец сил. Отличие состоит только в том, что знаки амплитуд кривых меняются местами при тех же фазе опорного сигнала и исходном состоянии азимута поля волны.

То обстоятельство, что в диапазоне линейности закона Гука, действующего в кристалле Si практически во всем его диапазоне прочности, деформации суммируются, позволяет нам вычитанием кривых рис. 3 получить напряжение, индуцированное тепловым потоком. Его координатное распределение показано на рис. 4, $a$, из которого можно сделать вывод о том, что кривая, его изображающая, обладает в пределах $1.5<x<4.5$ практически постоянной кривизной. Основанием для такого вывода является тот факт, что в установившемся состоянии функциональная связь потенциала $u$ (температуры) с напряжением $\sigma$ описывается уравнением Пуассона [12] $\partial^{2} u / \partial x^{2}=-\sigma / \tau$, где $1 / \tau-$ коэффициент пропорциональности. Следовательно, двойным графическим интегрированием координатной функции напряжения при использовании соответствующих граничных условий можно получить распределение температуры. Результат моделирования согласно изложенной процедуре приведен на рис. $4, b$. В качестве первого граничного условия здесь использована постоянная составляющая $C$ в температурной функции $u(x)$, величина которой определена подгонкой для выполнения второго условия установления границ интегрирования с реальным перепадом температур. Как показано в [13] на примере термоупругости в стекле от одномерного теплового потока, результат моделирования удовлетворительно согласуется с температурной функцией, полученной независимым методом. В данном случае такая возможность также может быть осуществлена, например, с помощью радиометра, что не является принципиальным. Важно другое, что величина напряжения и его локализация связаны не с абсолютным значением температуры, а с кривизной температурной функции, что имеет место на рис. $4, b$.

Что же касается функции $\sigma(x)_{I=0}$, то ее рассмотрение также возможно в рамках проделанного моделирования. Однако в качестве аналога потенциала здесь нужно использовать поверхностную энергию, неоднородность которой связана прежде всего со сложным
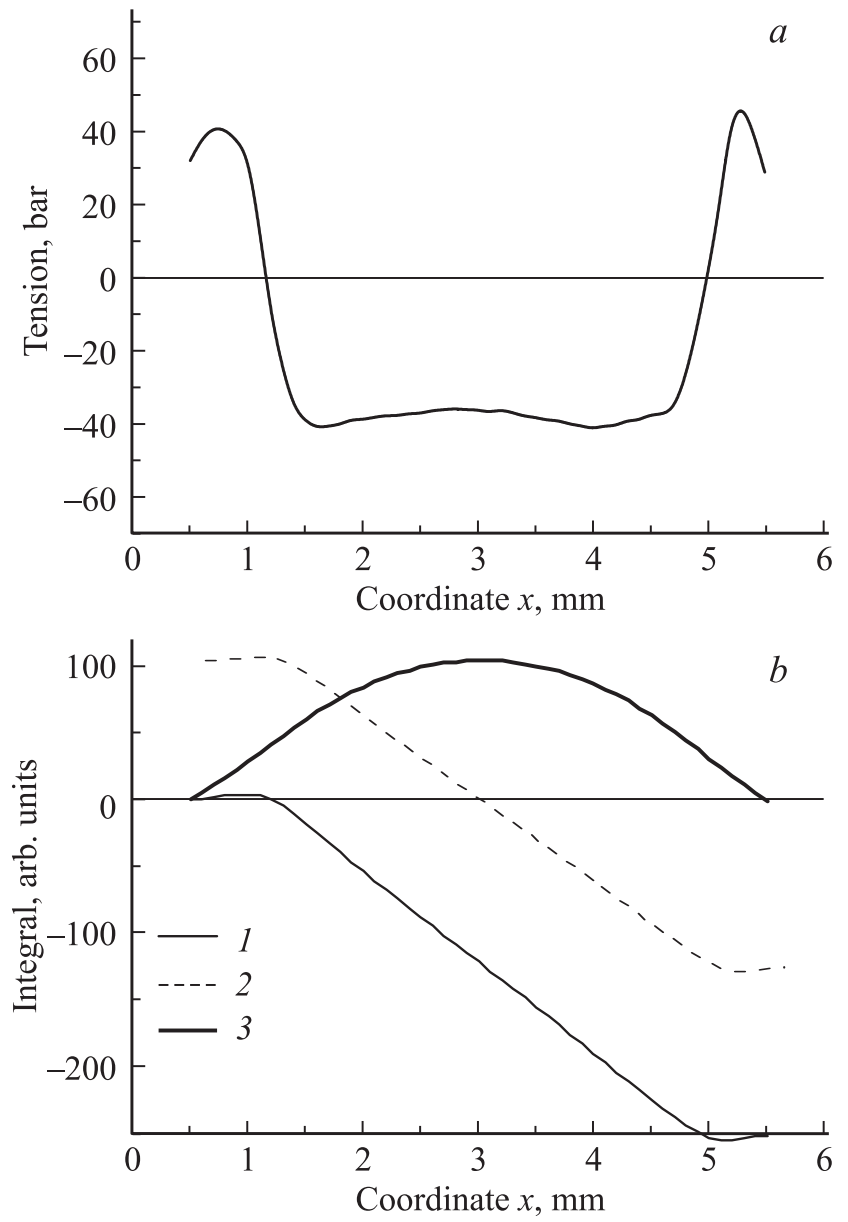

Рис. 4. Координатные зависимости: $a-$ разности термических напряжений $\sigma(x)_{I=0}-\sigma(x)_{I>0} ; b-$ интеграл $\sigma(1)$, интеграл функции $\sigma(x)+C(2)$, учитывающей постоянную составляющую, теряющуюся при первой операции дифференцирования, второй интеграл функции $\sigma(x)+C(3)$.

профилем структуры. В виде проекции на координату $x$ эта неоднородность может индуцировать напряжение, исследование которого является отдельной задачей микромеханики.

\section{4. Заключение}

В микромеханическом приборе, которым является кремниевый интегральный преобразователь давления, рассмотрена задача определения природы, величины и координатной локализации механического напряжения. В силу специфики объекта исследования задача решается техникой модуляционной поляриметрии зондирующего излучения. Ее главная особенность состоит в высокой, свойственной интерференционным устройствам, чувствительности к изменению состояния поляризации излучения, возникающему при его взаимодействии с образцом. Большой вследствие этого динамический диапазон измеряемой величины является информативным источником, из которого после проведенной соответ- 
ствующим образом обработки получены сведения о причинах возникновения напряжений, их величине и пространственном распределении. Изложенные результаты исследований могут быть использованы в диагностике кристаллов и структур на предмет величин не только внутренних остаточных напряжений, но и напряжений, индуцированных некоторыми технологическими воздействиями - операцией сборки в корпус, номинальным давлением, режимами электропитания.

\title{
Список литературы
}

[1] W. Erler, L. Walter. Elektrischen messen nichtelektrischen großen mit halbleiterwiderstanden (Verlag Technik, Berlin, 1973).

[2] В.М. Стучебников. Измерения, контроль, автоматизация, № 1 (45), 30 (1983).

[3] И.П. Жадько, В.А. Романов, Б.К. Сердега. ПТЭ, № 5, 215 (1972) [А.c. № 401291, Бюл. изобретат., № 29 (1977)].

[4] П.П. Поливанов. Изв. вузов. Радиоэлектроника, 24 (12), 93 (1978).

[5] R. Allan. Electronics, 53 (24), 113 (1980).

[6] В.И. Ваганов, Н.И. Гончарова. Изв. вузов. Электроника, № 1, 85 (1980).

[7] L.B. Wilner. ISA Trans., 17 (1), 83 (1978).

[8] Г.С. Ландсберг. Оптика (М., ГИТТЛ, 1957).

[9] Б.К. Сердега. Патент № 19983А (Украина). Опубл. 01.07.1994, Бюл. № 6.

[10] A. Gerrard, J.M. Burch. Introduction to matrix methods in optics (London, 1975).

[11] B.K. Serdega, Ye.F. Venger, Ye.V. Nikitenko. Semicond. Phys. Quant. Electron. Optoelectron., 2 (2), 153 (1999).

[12] А.Д. Коваленко. Основы термоупругости (Киев, Наук. думка, 1970).

[13] И.Е. Матяш, И.Е. Минайлова, О.Н. Мищук, Б.К. Сердега. ФTT, 55 (5), 1003 (2013).

Редактор Л.В. Шаронова

\section{Modulation-polarization diagnostics of thermal stresses in the integrated pressure transducer}

\author{
I. Mikhailenko ${ }^{1}$, A. Orlov ${ }^{2}$, B. Serdega ${ }^{1}$ \\ ${ }^{1}$ Lashkaryov Institute of Semiconductor Physics, \\ National Academy of Sciences of Ukraine, \\ $03028 \mathrm{Kiev}$, Ukraine \\ ${ }^{2}$ National Technical University of Ukraine \\ „Kyiv Polytechnic Institute“, \\ 03057 Kiev, Ukraine
}

\footnotetext{
Abstract A semiconductor pressure transducer, consisting of a sensing resistor implanted in resilient mechanical element formed as a silicon membrane, was analyzed by the method of modulation polarimetry. Coordinate distribution of uniaxial residual stress correlated with local inhomogeneities of the crystal, caused by its doping and heating by flowing current. Temperature distribution of the heat caused by the current in the resistor was obtained by dual integration of the pressure function, adjusted for relevant boundary conditions.
} 